\title{
Full recovery from severe SARS-CoV-2 infection after requiring extracorporeal membrane oxygenation and continuous hemodiafiltration
}

\section{Takafusa Yoshioka}

Tokyo Metropolitan Geriatric Hospital and Institute of Gerontology

Hiroshi Koganemaru

Tokyo Metropolitan Geriatric Hospital and Institute of Gerontology

\section{Tomohiro Murata}

Tokyo Metropolitan Geriatric Hospital and Institute of Gerontology

\section{Akiko Mano}

Tokyo Metropolitan Geriatric Hospital and Institute of Gerontology

\section{Kazuma Date}

Tokyo Metropolitan Geriatric Hospital and Institute of Gerontology

\section{Mitsuhiro Kawata}

Tokyo Metropolitan Geriatric Hospital and Institute of Gerontology

\section{Kyoko Komatsu}

Tokyo Metropolitan Geriatric Hospital and Institute of Gerontology

Hajime Fujimoto

Tokyo Metropolitan Geriatric Hospital and Institute of Gerontology

Hiroshi Yamamoto

Tokyo Metropolitan Geriatric Hospital and Institute of Gerontology

Nanae Aritaka

Toshima Hospital, Tokyo Metropolitan Health and Hospitals Corporation

\section{Masahiro Sano}

Toshima Hospital, Tokyo Metropolitan Health and Hospitals Corporation

\section{Takuya Adachi}

Toshima Hospital, Tokyo Metropolitan Health and Hospitals Corporation

Masaki Anraku ( $\sim$ anraku-ths@umin.ac.jp)

Tokyo Metropolitan Geriatric Hospital and Institute of Gerontology

\section{Case Report}

Keywords: COVID-19, SARS-CoV-2, extracorporeal membrane oxygenation, hemodiafiltration 
Posted Date: August 28th, 2020

DOl: https://doi.org/10.21203/rs.3.rs-65591/v1

License: (c) (i) This work is licensed under a Creative Commons Attribution 4.0 International License. Read Full License 


\section{Abstract}

We report two cases of severe SARS-CoV-2 infection successfully treated with venovenous extracorporeal membrane oxygenation (VV-ECMO) and continuous hemodiafiltration (CHDF). ECMO was indicated despite immediate anti-viral and anticoagulation therapy with maximal ventilatory support. Meticulous bronchoscopic drainage was necessary during treatment by ECMO due to copious fluid secretion. Renal replacement therapy with continuous hemodiafiltration was also required for both cases due to ongoing renal dysfunction. However, both patients recovered fully without any disability or neurological sequelae. VV-ECMO is a vital tool to maintain appropriate gas exchange for severe SARS-CoV-2 infection to facilitate functional recovery. Because subsequent renal impairment is common in such severe cases, hemodiafiltration undertaken in a timely manner may be necessary. Controlling secondary bacterial infection is also key for lung recovery, as it may otherwise compromise the chance to wean patients off ECMO.

\section{Introduction}

Severe acute respiratory syndrome coronavirus 2 (SARS-CoV-2) can cause fatal damage primarily to the lung, but also to the kidney. Here, we report two cases of successful recovery from severe SARS-CoV-2 infection which had necessitated multiple organ replacement interventions by veno-venous extracorporeal membrane oxygenation (VV-ECMO) and continuous hemodiafiltration (CHDF).

\section{Case Presentation}

Case 1. A 54 year-old obese male ex-smoker with high fever, general fatigue, and loss of appetite for a week was proven positive for SARS-CoV-2 by polymerase chain reaction (PCR) testing. Clinical characteristics are summarized in Table 1. Despite antiviral therapy with favipiravir, his respiratory condition deteriorated rapidly and he required mechanical ventilation in a negative-pressure room soon after hospital admission. Despite high positive end-expiratory pressure (PEEP, $15 \mathrm{~cm} \mathrm{H}_{2} \mathrm{O}$ ) and highpressure support $\left(20 \mathrm{~cm} \mathrm{H}_{2} \mathrm{O}\right.$ ) with $100 \%$ oxygen, poor oxygenation could not be remedied; partial pressure of oxygen $\left(\mathrm{pO}_{2}\right)$ was only $91.9 \mathrm{~mm} \mathrm{Hg}$ with hypercapnia $\left(\mathrm{pCO}_{2}, 47.8 \mathrm{~mm} \mathrm{Hg}\right)$. The patient was hemodynamically stable with preserved cardiac function. VV-ECMO was introduced without any procedure-related complications (Table 1). Computed tomography (CT) images on admission revealed bilateral thick and patchy infiltrates (Fig. 1a, b). Intravenous unfractionated heparin infusion was started to prevent possible thromboembolism because of the known hypercoagulable status of SARS-CoV-2infected patients. Nafamostat mesylate $(0.2 \mathrm{mg} / \mathrm{kg} / \mathrm{hr})$, a serine protease inhibitor, was also used as it is proposed to inhibit viral entry into the cytoplasm. Muscle relaxation was realized by continuous infusion of rocuronium bromide (for muscle blockade). Broad-spectrum antibiotic treatment (piperacillin/tazobactam) was initiated followed by vancomycin to cover concomitant MRSA pneumonia. Although the bilateral lungs were totally whited out, antibiotic therapy with meticulous bronchoscopic drainage could effectively recover lung function. Because the patient tolerated weaning off ECMO successfully, and this was terminated after an 11-day run (Table 2). The patient was extubated 6 days 
after ECMO cessation (Fig. 1c, d). CHDF was initiated 2 days thereafter ECMO, but could be terminated after hospital transfer out of the ICU. There were no major ECMO-related complications during the clinical course. Magnetic resonance imaging of the brain after extubation did not reveal any signs of thromboembolism, bleeding, or degenerative changes. The patient was finally discharged from the hospital with almost full functional recovery without any disability.

Case 2. A 60 year-old male current smoker was transferred to our hospital 10 days after the onset of a high fever (Table 1). PCR testing confirmed SARS-CoV-2 infection. VV-ECMO was started immediately because of uncompensated acidosis $(\mathrm{pH}$ 7.19) with poor oxygenation (a P/F ratio of 103) despite maximal ventilatory support with $100 \%$ oxygen (Fig. 2a, b). His hemodynamic status required fluid replacement during ECMO initiation, but cardiac function was preserved. Favipiravir was given for 7 days before ECMO was started, and switched to Remdesivir thereafter. Intravenous unfractionated heparin, nafamostat mesylate $(0.2 \mathrm{mg} / \mathrm{kg} / \mathrm{hr})$, and rocuronium bromide were also given continuously during ECMO. Airway bleeding occurred on the day following ECMO initiation, but soon subsided after discontinuing nafamostat mesylate. Bacterial infection of the emphysematous lungs required broadspectrum antibiotic therapy (piperacillin/tazobactam and vancomycin), meticulous bronchoscopic drainage, and prone positioning on VV-ECMO. This case needed tracheostomy together with prolonged mechanical ventilator support due to the underlying lung condition. After intensive rehabilitation, the patient was successfully weaned off mechanical ventilation, and recovered fully with no neurological sequelae. The patient was transferred from the ICU for further rehabilitation without oxygen support (Fig. 2c). Contrast computed tomography of the brain revealed several micro-lesions suspected as being due to thrombotic or infectious emboli in the cortex, but they did not translate into any recognizable symptoms (Fig. 2d).

\section{Discussion}

In their interim guidelines, the World Health Organization (WHO) stated that SARS-CoV-2-related acute respiratory distress syndrome (ARDS) could be treated by ECMO intervention in expert centers. ${ }^{1}$ According to the international registry, more than 2,000 cases of SARS-CoV-2 ARDS have been treated by ECMO, more than $90 \%$ of which were in the VV mode. ${ }^{2}$ Aside from critical issues related to limited numbers of devices and human resources in the recent COVID-19 pandemic, the role of ECMO appeared to be favored as a vital tool for bridging the patient to recovery from severe ARDS. However, indications for its use have to be carefully assessed, and long-term outcomes need to be monitored. Once ECMO is deemed indicated, timely introduction is preferable to allow injured lungs to rest and recover as much as possible, because of its ability to confer lung protection from mechanical ventilator-associated injury. ${ }^{3}$

Among the contraindications for ECMO administration, obesity (BMI of 35.6 in Case 1) and underlying lung disease (emphysema in Case 2) require extra care over the course of treatment. Bilateral diaphragmatic elevation by the obese abdomen makes it difficult to maintain efficient ventilatory volume. Prone positioning, proven to be effective for ARDS, ${ }^{4}$ was not undertaken for Case 1 because it could not be performed safely with ECMO cannulas in place in this severely obese patient. In Case 2, we performed 
early tracheostomy so that the patient could be awake and mobilized for rehabilitation on a ventilator. The patient fortunately recovered with preserved lung function requiring no oxygen therapy. Frequent bronchoscopic drainage to maintain the airway was required in both cases with maximal precautions regarding the use of personal protective equipment by the medical staff.

Among extrapulmonary injuries of severe SARS-CoV-2 infection, renal impairment was observed in both cases presented here, consistent with plausible mechanisms already described, such as direct viral infection by engaging angiotensin-converting enzyme 2 receptors with co-expressed transmembrane serine proteases triggering renal endothelial damage. ${ }^{5}$ Multiple antibiotics, extracorporeal circulation, and transient perfusion imbalance might also have contributed to causing decreased renal function. Great care was taken to maintain end-organ perfusion with appropriate oxygen delivery, management of which might minimize the duration of temporary hemodiafiltration. Coagulopathy, most likely a consequence of a profound inflammatory response, can cause massive thrombosis in critically ill SARS-CoV-2 cases. ${ }^{6}$ In a French multicenter study with a propensity-score matching analyses, comparison with non-SARS-CoV-2 ARDS revealed that significantly more thromboembolic events (mainly pulmonary embolisms) were seen in patients with SARS-CoV-2-related ARDS (4.8\% versus $11.7 \%) .{ }^{7}$ In the current cases, clinically evident thromboembolic events were not seen, perhaps because of the early introduction of anticoagulation therapy along with nafamostat mesylate (a serine protease inhibitor) treatment which might also contribute to reducing thromboinflammation. 8,9

In summary, SARS-CoV-2 ARDS could be treated successfully by timely introduction of organ replacement therapy using VV-ECMO and hemodiafiltration. Long-term follow-up of patients who survive SARS-CoV-2 ARDS is warranted, because sequelae of severe SARS-CoV-2 infection remain unclear.

\section{Declarations}

Consent: The patients consented to publish their case, data and accompanying images.

Source of funding: None

Conflict of interest: The authors declare that they have no conflict of interest.

\section{References}

1. World Health Organization. Clinical management of COVID-19 Interim guidance, published 27 May 2020. https://www.who.int/publications/i/item/clinical-management-of-severe-acute-respiratoryinfection-when-novel-coronavirus-(ncov)-infection-is-suspected Accessed August 3, 2020

2. The Extracorporeal Life Support Organization (ELSO): ECMO in COVID-19. https://www.elso.org/Registry/FullCOVID19RegistryDashboard.aspx Accessed August 1, 2020

3. Shekar, K. et al. Extracorporeal Life Support Organization Coronavirus Disease 2019 Interim Guidelines: A Consensus Document from an International Group of Interdisciplinary Extracorporeal 
Membrane Oxygenation Providers. ASAIO J. 66, 707-721 (2020).

4. Guérin C, Reignier J, Richard JC, et al. Prone positioning in severe acute respiratory distress syndrome. N Engl J Med. 2013;368(23):2159-2168.

5. Gupta A, Madhavan M V., Sehgal K, et al. Extrapulmonary manifestations of COVID-19. Nat Med. Published online July 1, 2020. doi:10.1038/s41591-020-0968-3

6. Zhang Y, Xiao M, Zhang S, et al. Coagulopathy and antiphospholipid antibodies in patients with covid-19. N Engl J Med. 2020;382(17):e38.

7. Helms J, Tacquard C, Severac F, et al. High risk of thrombosis in patients with severe SARS-CoV-2 infection: a multicenter prospective cohort study. Intensive Care Med. Published online 2020. doi:10.1007/s00134-020-06062-x

8. Doi $K$, lkeda $M$, Hayase $N$, et al. Nafamostat mesylate treatment in combination with favipiravir for patients critically ill with Covid-19: a case series. Crit Care. 2020;24(1). doi:10.1186/s13054-02003078-z

9. Connors JM, Levy JH. Thromboinflammation and the hypercoagulability of COVID-19. J Thromb Haemost. 2020;18(7):1559-1561.

\section{Tables}

Table 1. Clinical characteristics on admission.

\begin{tabular}{|c|c|c|c|c|c|c|c|}
\hline Case & Age/gender & Height/weight/BMI & SOFA score & Co-infection & Comorbidities & $\begin{array}{l}\text { Onset to ECMO* } \\
\text { (days) }\end{array}$ & $\begin{array}{l}\text { D-dimer } \\
(\mu g / m l)\end{array}$ \\
\hline 1 & 54/Male & $175 \mathrm{~cm} / 109 \mathrm{~kg} / 35.6$ & 4 & MRSA & $\begin{array}{l}\text { Diabetes mellitus } \\
\text { Hypertension }\end{array}$ & 8 & 1.8 \\
\hline 2 & 60/Male & $170 \mathrm{~cm} / 75 \mathrm{~kg} / 26.0$ & 4 & $\begin{array}{l}\text { H. influenzae } \\
\text { MSSA }\end{array}$ & $\begin{array}{l}\text { Diabetes mellitus } \\
\text { Emphysema }\end{array}$ & 10 & 4.6 \\
\hline
\end{tabular}

SOFA, sequential organ failure assessment; MRSA, Methicillin-resistant Staphylococcus aureus; MSSA, methicillin-susceptible Staphylococcus aureus; H. Influenzae, Haemophilus influenzae.

* Duration between the day of symptom onset and ECMO administration

Table 2. Summary of ECMO support 


\begin{tabular}{|c|c|c|c|c|c|c|}
\hline Case & Mode of ECMO & Cannulation site (size, Fr) & ECMO system & $\begin{array}{l}\text { ECMO flow }(\mathrm{L} / \mathrm{min}) / \\
\text { Pump rotation (rpm) }\end{array}$ & ECMO-run (days) & Complication \\
\hline 1 & Venovenous & $\begin{array}{l}\text { Right femoral vein }(25) \\
\text { Right internal jugular vein }(21)\end{array}$ & EMERSAVE $^{\circledR}$ & $5-6 / 2,000-2,300$ & 11 & None \\
\hline 2 & Venovenous & $\begin{array}{l}\text { Right femoral vein (24) } \\
\text { Right internal jugular vein }(21)\end{array}$ & EMERSAVE $^{\circledR}$ & $5-6 / 2,000-2,500$ & 11 & Airway bleeding \\
\hline
\end{tabular}

\section{Figures}

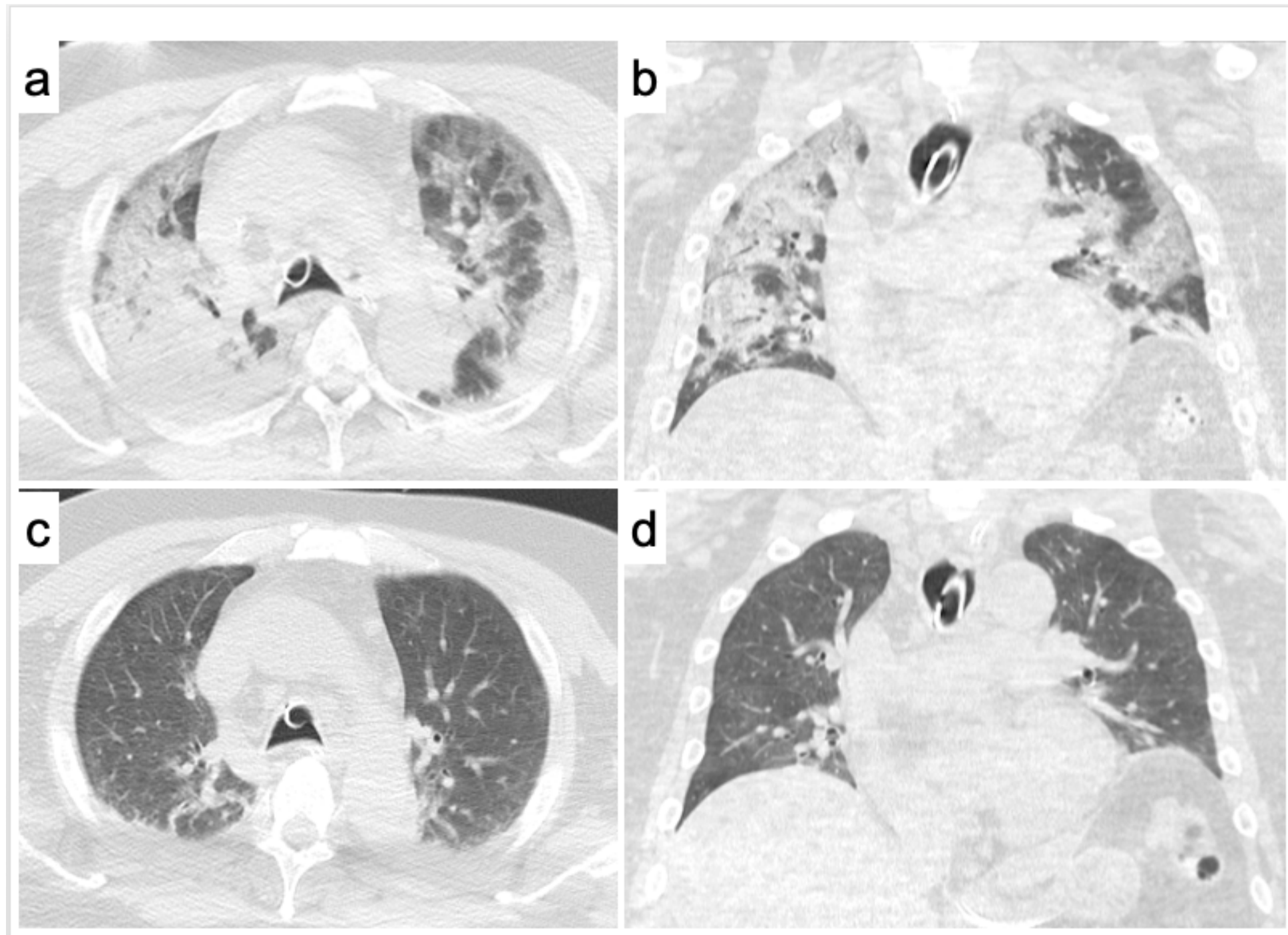

\section{Figure 1}

Case 1. Computed tomography demonstrating diffuse bilateral consolidation and infiltrates in axial (a) and coronal (b) images. Infiltration resolved with no signs of lung fibrosis in axial (c), and coronal (d) images after VV-ECMO and mechanical ventilatory support. 


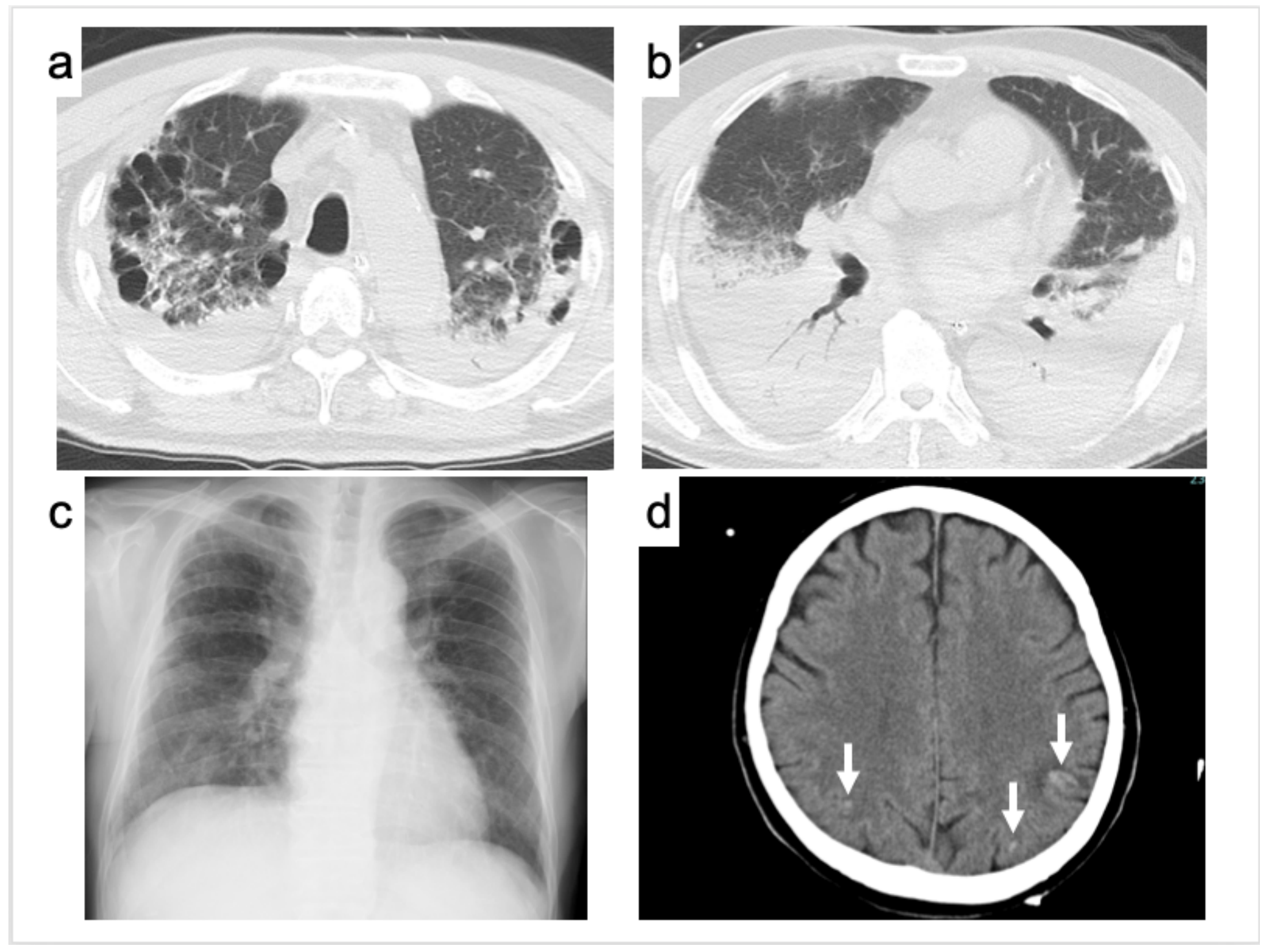

Figure 2

Case 2. Computed tomography (CT) of the chest depicting bilateral, dorsally-predominant consolidation of the lungs with underlying emphysematous changes $(a, b)$. Chest $X$-ray taken before discharge showing full recovery from the severe lung injury (c). CT of the brain showing multiple lesions in the cortex of the brain suspected to be multiple thrombotic or infectious emboli (arrows, d). 